Proc. of the XXVII Intern. School on Physics of Semiconducting Compounds, Jaszowiec 1998

\title{
FROM MAGNETIC POLARONS TO FERROMAGNETISM
}

\author{
T. DietL \\ Institute of Physics, Polish Academy of Sciences and College of Science \\ al. Lotników 32/46, 02-668 Warszawa, Poland
}

\begin{abstract}
A brief overview is given on selected effects of the exchange coupling between effective mass electrons and localized spins in II-VI semiconductors containing $\mathrm{Mn}$ ions. In the case of a carrier or exciton trapped by an impurity or defect, the exchange interaction leads to zero-field spin-splitting. The current theory of such complexes, known as bound magnetic polarons, describes correctly their spectroscopic and thermodynamic properties as well as their formation dynamics. At the same time, a free magnetic polaron a delocalized carrier accompanied by a traveling cloud of polarized spins is not expected to exist for the actual values of the coupling constants. However, hole scattering by thermodynamic and static fluctuations is shown to affect significantly its energy. For a strong coupling, the corresponding renormalization has to be described by a non-perturbative approach. Finally, the influence of the carrier liquid upon the Mn spins is discussed. Here, either optical pumping or $p$-type doping may lead to a ferromagnetic order, both in bulk and layered structures. Because of a long-range character of the carrier mediated interactions, this ordering is not destroyed by the fluctuations, even in the reduced dimensionality systems.
\end{abstract}

PACS numbers: 78.47.+p, 75.70.Cn, 78.55.Et, 78.66.Hf

\section{Introduction}

In this paper, selected theoretical and experimental results concerning effects of the exchange coupling between effective mass electrons and localized spins are reviewed. While in diluted magnetic metals the main signatures of such a coupling is the Kondo effect and the oscillatory Ruderman-Kittel-Kasuya-Yosida (RKKY) interaction, a number of other novel effects has been put into the evidence and examined in detail by many groups in semimagnetic semiconductors. In the first part of the paper, the questions concerning physics of bound and free magnetic polaron are addressed. The renormalization of the carrier energy by spin and chemical disorder is then discussed. Finally, the influence of the carrier liquid upon the localized spins is described. The discussion is limited to II-VI semiconductors containing $\mathrm{Mn}$ ions, and the review is by no means exhausting. It aims merely at recalling some of the topics which have attracted considerable attention over the recent twenty years or so. A more complete account of the results and papers published prior to 1992 can be found in books devoted to semimagnetic semiconductors [1]. 


\section{Bound magnetic polarons}

Bound magnetic polaron (BMP) [2], that is a cloud of magnetic ions ferromagnetically ordered by the exchange interaction with an effective mass carrier in a localized state, has become one of the central theme in the physics of Mn-based semimagnetic semiconductors. Indeed, the polaron effects determine - to a large extent - optical, transport, and thermodynamic properties of bulk [3] and layered semiconductor structures [4] containing localized spins.

The presence of magnetic polarons in semimagnetic semiconductors was first evidenced by noting [5] that the binding energy of an exciton trapped by a neutral acceptor $A^{0} X$ in $\mathrm{Cd}_{1-x} \mathrm{Mn}_{x} \mathrm{Te}$ increased strongly with lowering temperature. This observation stimulated a number of detailed experimental works, from which particularly rewarding were studies of donor states by Raman spectroscopy [6-11]. This is so because the cross-section for spin-flip Raman scattering (SFRS) is directly proportional to the spin-spin correlation function of the donor electron, a quantity which could be analytically calculated, even with the effect of thermodynamic fluctuations of magnetization taken into account $[12,13]$. The important role of the fluctuations stems from the finite volume involved, which precludes the existence of any second order phase transition [12,14]. Actually, as shown in Fig. 1, the fluctuations give the dominant contribution to the donor spin-splitting at high temperatures. They determine also the width and shape of the SFRS line, as shown in Fig. 2. The same model [12] describes quantitatively thermodynamic properties such as the BMP contribution to the impurity activation energy [15], depicted in Fig. 3, and magnetic susceptibility [16], presented in Fig. 4.
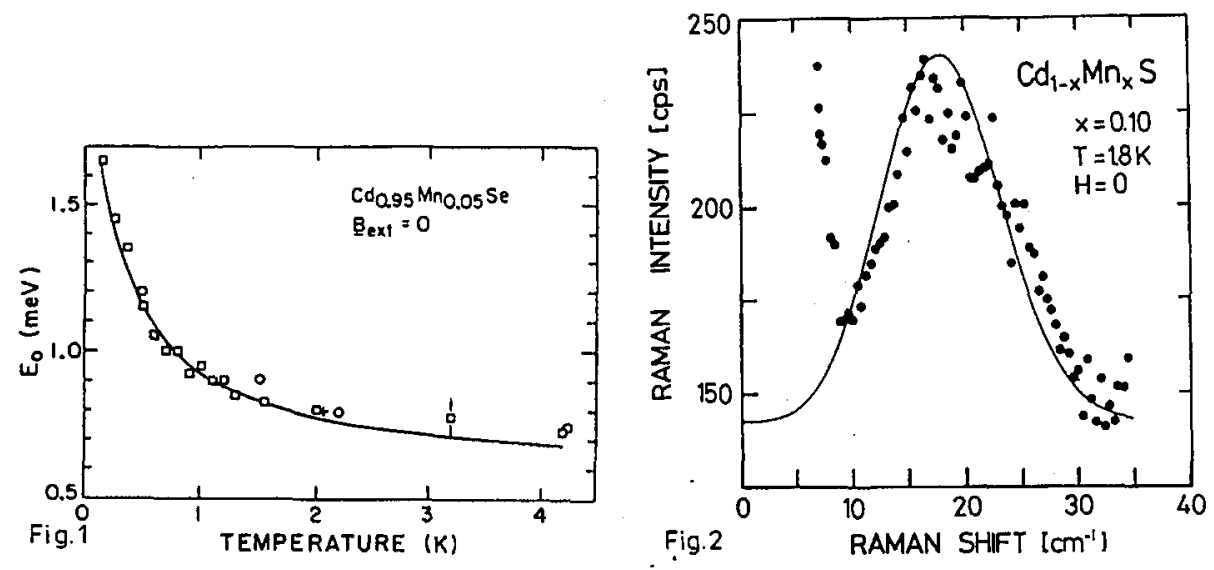

Fig. 1. Zero-field spin-splitting of the donor level as measured by spin-flip Raman scattering (circles [6], plus [9], squares [11]). According to the theory [12] (solid line), the spin-splitting below $1 \mathrm{~K}$ is dominated by the effect of magnetic polaron, while above - by the fluctuations of magnetization (after [11]).

Fig. 2. Spin-flip Raman scattering spectrum of donor electrons [10] compared to the theoretical predictions (solid line) for the bound magnetic polarons [12] (after [10]). 


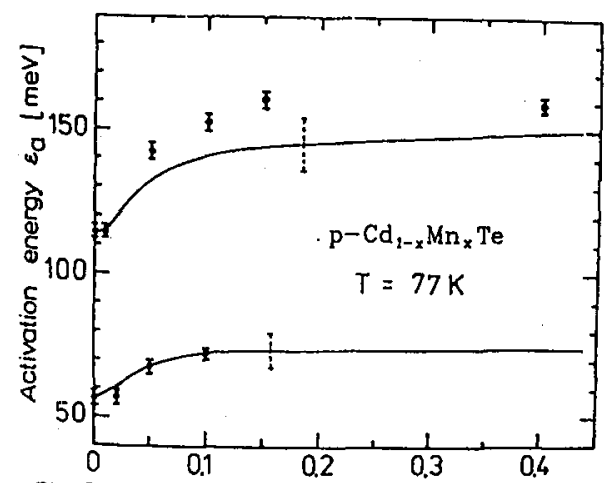

Fig. 3

Composition $x$

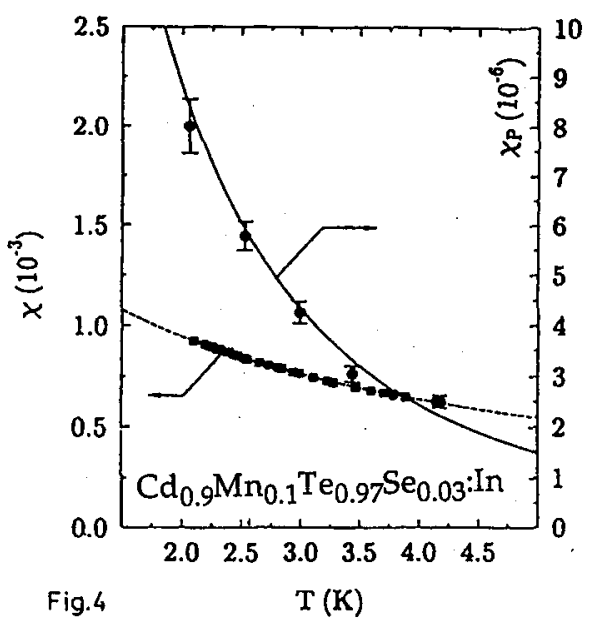

Fig. 3. Activation energy of electric conductivity for two different acceptors compared to the theory (solid lines) for the bound magnetic polarons (after [15]).

Fig. 4. Experimental total magnetic susceptibility $\chi$ and a contribution from the bound magnetic polarons $\chi_{\mathrm{p}}$ [16] compared to the theoretical expectations [12] shown by solid line (after [16]).

Another important line of research started with the observation of polaronic effects in exciton luminescence [17], which could not be associated with an exciton trapped by an occupied impurity. An important problem addressed by this observation was that the organization of the localized spins under the influence of the exchange field of the exciton spin had to proceed rather fast, faster than the exciton recombination. A recent rapid progress in the application of ultrashort light pulses for monitoring dynamic processes has made it possible to trace directly the emerging and vanishing of polarization of the localized spins after optical injection of carriers across the band gap in bulk [18-24] and layered [25-29] semimagnetic semiconductors.

In order to discuss the dominant microscopic mechanisms that control polaron dynamics, it is useful to refer to Fig. 5 [30], where the polaron formation rate $\tau_{\mathrm{f}}^{-1}$, as determined by various authors [21-24], is compared to the experimentally obtained $[31,32]$ spin-lattice $\tau_{\mathrm{SL}}^{-1}$ and the spin-spin relaxation rate $\tau_{\mathrm{SS}}^{-1}$, deduced from the EPR studies [33,34]. As shown, $\tau_{\mathrm{f}}$ deduced from time-resolved luminescence [21-23, 27, 28] and from the SQUID magnetometry [24] are of the same order. This suggests [24] that spin diffusion [20,35] does not give the dominant contribution to the polaron formation, as the diffusion conserves the total magnetic moment and, therefore, changes of the local magnetization by such a process are not visible in the time-resolved magnetometry. Furthermore, spin-lattice relaxation is by more than two orders of magnitude too slow to lead to a picosecond timescale of polaron formation. Actually, because of shrinkage of the polaron volume during its formation, $\tau_{\mathrm{f}}$ is expected to be even longer than the relevant relaxation time of the spin subsystem $[30,36]$. At the same time, $\tau_{f}$ agrees very 


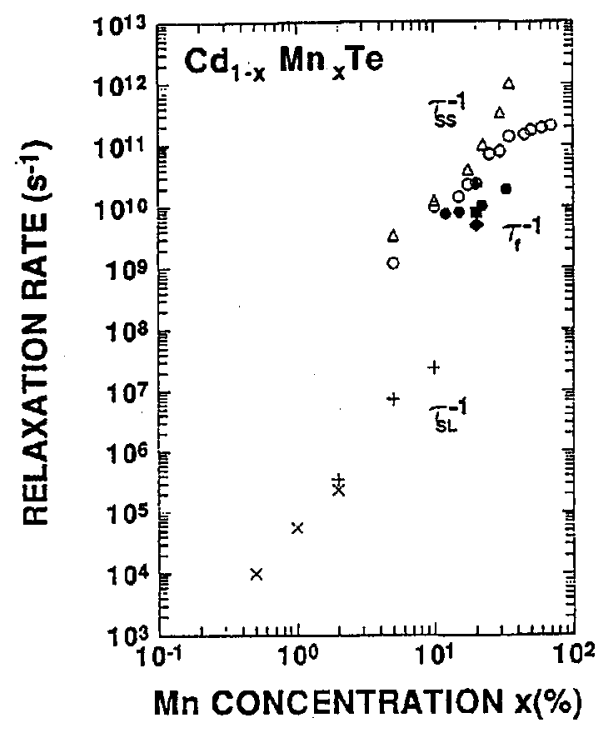

Fig. 5. Spin-lattice relaxation rate at $5 \mathrm{~K}$ (crosses [31] and pluses [32]) and spin-spin relaxation rate (empty circles [33] and triangles [34]) compared to polaron formation rate (full triangles [21], squares [22], circles [23] - time-resolved luminescence, and diamonds [24] — time-resolved magnetization) (after [30]).

well with the spin-spin relaxation time $\tau_{\mathrm{SS}}$ deduced from the EPR studies $[33,34]$. This appears to be rather striking as the polaron formation involves both spin and energy relaxations.

In order to explain why the spin response to the high-frequency magnetic field is described by $\tau_{\mathrm{SS}}$, not by $\tau_{\mathrm{SL}}$, it is instructive to recall that there is no spin response to the external field for $t<\tau_{\mathrm{SL}}$ if spin-spin interactions are of the Heisenberg form, so that the magnetic moment is a constant of motion and $\tau_{\mathrm{SS}}^{-1}=0$. If, however, the non-scalar part of the spin-spin interactions is strong enough, a non-zero magnetization can be adiabatically formed already at $\tau_{\mathrm{SL}}>$ $t \geq \tau_{\mathrm{SS}}[30,31,37,38]$. Thus, the conservation of the spin momentum is broken by strong non-scalar spin-spin interactions which shift the spin-response time down to the picosecond range, while the relaxation of the photocarrier energy occurs adiabatically at the expense of all kinds of the interactions among the localized spins.

\section{Free and self-trapped magnetic polarons}

Despite some experimental suggestions and theoretical models, a consensus is emerging that a free magnetic polaron, a delocalized carrier accompanied by a traveling cloud of polarized spins, is not expected to exist in semimagnetic semiconductors. This is because coherent tunneling of quasi-particles dressed by spin polarization is hampered, in disordered magnetic systems, by a small quantum overlap between magnetizations in the different space regions. 
At the same time, the observation of the exciton magnetic polaron addresses a question about the conditions under which self-trapping of an effective mass carrier by localized spins could occur. While some theoretical works [39] prepared the ground, the point was made by Benoit à la Guillaume [14], who showed that self-trapping of effective mass carriers by the exchange interaction with localized spins is not possible in three- and two-dimensional systems for the actual material parameters. The observations of excitonic magnetic polarons imply therefore the presence of an additional localizing potential. This conclusion is confirmed by direct calculations $[30,36]$, which provide the magnitude of the prelocalizing potential, necessary to explain the experimental values of the polaron contribution to the exciton energy. This potential appears to be merely brought about by alloy fluctuations, a conjecture strongly supported by a much greater value of the exciton polaron energy in $\mathrm{Cd}_{1-x-y} \mathrm{Mn}_{x} \mathrm{Mg}_{y} \mathrm{Te}$ than in $\mathrm{Cd}_{1-x} \mathrm{Mn}_{x} \mathrm{Te}$ [23].

\section{Beyond molecular-field and virtual-crystal approximations}

A growing amount of experimental results demonstrates how scattering of carriers by thermodynamic and frozen fluctuations affects their energy and spin lifetime without, however, leading to the spin-splitting in the absence of an external magnetic field. If the depth of the potential well introduced by the transition metal

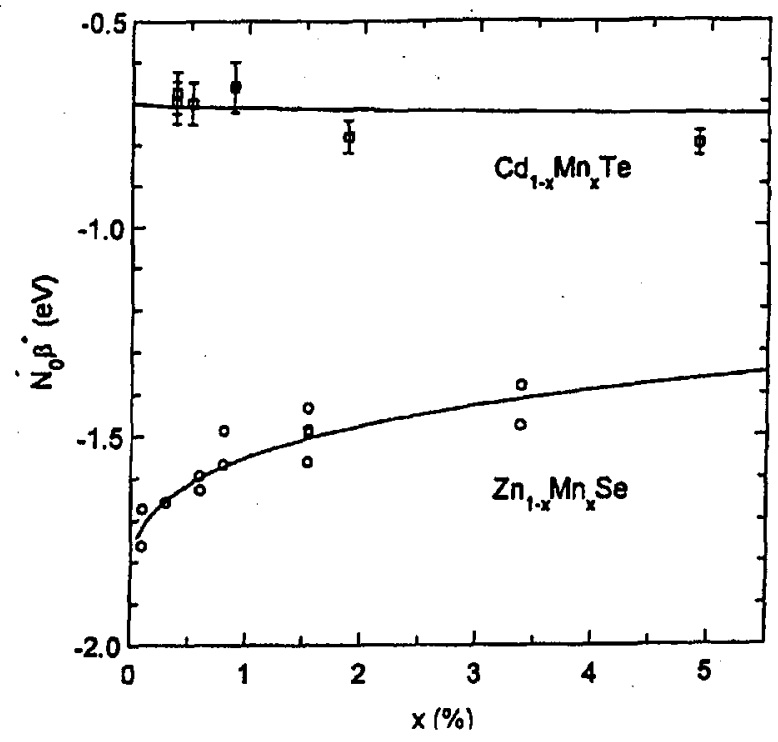

Fig. 6. Apparent exchange integral of the valence band as determined [45] in the framework of the molecular-field and virtual-crystal approximations from the ratio of field-induced exciton splitting to magnetization, compared to the diagrammatic alloy theory [42] (solid lines) for repulsive $\left(\mathrm{Cd}_{1-x} \mathrm{Mn}_{x} \mathrm{Te}\right)$ and attractive $\left(\mathrm{Zn}_{1-x} \mathrm{Mn}_{x} \mathrm{Se}\right)$ alloy potentials (reprinted from [45], Copyright (1998), with permission from Elsevier Science). 
is much smaller than the width of the relevant band, second-order perturbation theory can be applied in order to evaluate corrections to the molecular-field and virtual-crystal approximation [40]. By contrast, for large and attractive exchange and alloy potentials, such that the potential well associated with the transition metal is deep enough to almost form a bound state, the corrections have to be described by a non-perturbative approach [41]. In particular, a generalization of the alloy theory for the case of the spin-dependent potential [42] makes possible to describe quantitatively bowing of the energy gap, asymmetry of the spin splitting, and the dependence of the apparent hole exchange integral $\beta^{*}$ on the Mn concentration, as observed $[43,44]$ in $\mathrm{Cd}_{1-x} \mathrm{Mn}_{x} \mathrm{~S}$. A comparison of the dependence $\beta^{*}(x)$ in the case of $\mathrm{Zn}_{1-x} \mathrm{Mn}_{x}$ Se and $\mathrm{Cd}_{1-x} \mathrm{Mn}_{x} \mathrm{Te}$, where the total Mn potential acting on the hole is attractive and repulsive, respectively, is shown in Fig. 6 [45].

\section{Ferromagnetic transition induced by free carriers}

It has been known for a long time that the compensation of the intrinsic antiferromagnetic interactions by a ferromagnetic coupling would result in a dramatic enhancement of the sensitivity of semimagnetic semiconductors to the temperature and the magnetic field, particularly in the vicinity of the ferromagnetic phase transition. A search for the ferromagnetic transition has been successful in the case of Mn-based $p$-type IV-VI [46] and III-V compounds [47], in the latter case a critical temperature as high as $110 \mathrm{~K}$ has recently been reported for $\mathrm{Ga}_{0.95} \mathrm{Mn}_{0.05} \mathrm{As}[48,49]$. In the case of II-VI semimagnetic semiconductors, a tight binding model [50] suggests that the superexchange in Cr-based semimagnetic semiconductors might be dominated by a ferromagnetic contribution. Accordingly, an attempt has been undertaken [51] to overcome the well-known small solubility of $\mathrm{Cr}$ in II-VI compounds by means of MBE growth of $\mathrm{Cd}_{1-x} \mathrm{Cr}_{x} \mathrm{Te}$.

Recent progress in epitaxial doping of II-VI wide gap semiconductors by substitutional impurities stimulated a detailed analysis of the nature and strength of the spin-spin interactions mediated by itinerant electrons in bulk, layered, and nanostructured II-VI compounds [52]. For those cases, the dimensionality $d$ of the subsystem of the carriers is determined by the shape of the potential $V(\zeta)$ that leads to their confinement. Accordingly, the case $d=2$ and $d=1$ corresponds to a one- and two-dimensional potential well, respectively. It has been demonstrated that the RKKY approach, and the model, in which the mutual influence of the spin polarizations of the carriers and the magnetic ions are considered in a self-consistent way, lead to quantitative identical results in the mean field approximation (MFA). It has also been noted that this approximation should be valid in the case under consideration since, due to greater concentrations of the magnetic ion than those of the carriers, the RKKY interaction is essentially ferromagnetic over a long range in semimagnetic semiconductors. This suppresses the role of magnetization fluctuations, even in the reduced dimensionality systems. Accordingly, both the magnetic susceptibility and the derivative of the carrier spin-splitting, $\partial \Delta(T, H) / \partial H$, may undergo a critical divergence in the limit of vanishing magnetic fields and at a non-zero temperature $T_{\mathrm{c}}=\Theta-T_{0}$, where for a degenerate carrier liquid 


$$
\Theta=S(S+1) N_{0} I^{2} A_{\mathrm{F}} \rho_{\mathrm{d}}\left(\varepsilon_{\mathrm{F}}\right) \int \mathrm{d} \zeta \tilde{x}(\zeta)\left|\varphi_{0}(\zeta)\right|^{4} / 12 k_{\mathrm{B}}
$$

A similar model of ferromagnetism, developed for the case of a nondegenerate gas of carriers thermally activated from the impurity levels, was put forward by Pashitskii and Ryabchenko [53].

In the above formulae, temperature dependent $T_{0}>0$ and $\tilde{x}<x$ take the presence of antiferromagnetic interactions into account [54], $I \equiv \alpha$ or $\beta$ is the exchange integral of the relevant band, $A_{F} \geq 1$ is a Fermi liquid parameter that describes the enhancement of the RKKY interaction by the combined effect of carrier-carrier correlation and disorder [55], $\rho_{\mathrm{d}}$ is the total density of states at the Fermi level, which for one subband with a parabolic dispersion $\varepsilon(k)$ is given by $\rho_{\mathrm{d}}\left(\varepsilon_{\mathrm{F}}\right)=m^{*} k_{\mathrm{F}}{ }^{d-2} /\left[2^{d-2} \pi^{d / 2} \Gamma(d / 2) \hbar^{2}\right]$, and $\varphi_{0}(\zeta)$ is the envelope function of the relevant subband.

In the ferromagnetic regime, $T<T_{\mathrm{c}}$, the non-zero spin-splitting and magnetization of the $\mathrm{Mn}$ spins are determined by the magnitude of a self-consistent molecular field produced by the spin-polarized carriers.

Implicit in the model in question is an assumption, well-fulfilled in semimagnetic semiconductors, that the carrier bandwidth $V$ is greater than both the exchange energy $I N_{0}$ and the width of the $\mathrm{Mn}$ spin excitation spectrum. In such a case, each carrier interacts effectively with many Mn spins at the same time, and adjusts adiabatically its wave function to the instantaneous configurations of the $\mathrm{Mn}$ moments. This is in contrast to, e.g., ( $\mathrm{La}, \mathrm{Sr}) \mathrm{MnO}_{3}$-type materials, in which narrowness of the band makes the double exchange model to be better applicable than the RKKY approach. It is also worth noting that all material parameters conspire to make $T_{c}$ greater for the holes than for the electrons in a given structure of semimagnetic semiconductors. Actually, it has been predicted [52] that the condition for a ferromagnetic transition above $1 \mathrm{~K}, T_{\mathrm{c}} \geq 1 \mathrm{~K}$, can be met in the case of $p$-type II-VI semimagnetic semiconductors. Particularly promising in this context is recent progress in fabrication of quantum wells modulation-doped by nitrogen, in which weak disorder may preclude the formation of bound magnetic polarons.

Indeed, the presence of a ferromagnetic transition in a single, modulation-doped, 8-nm quantum well (QW) of $\mathrm{Cd}_{1-x} \mathrm{Mn}_{x} \mathrm{Te} / \mathrm{Cd}_{1-; ;}{ }_{-z} \mathrm{Mg}_{y} \mathrm{Zn}_{z} \mathrm{Te}: \mathrm{N}$ has been put into the evidence by observing colossal Zeeman splittings of interband optical transitions, probed by means of photoluminescence (PL) and its excitation spectra (PLE) $[56,57]$. The presence of the delocalized hole gas has been assessed by the Moss-Burstein shift between the energies of the PLE step and the PL maximum, according to which the hole concentration varies in the studied structures between ca. $1.5 \times 10^{11} \mathrm{~cm}^{-2}$ and $3 \times 10^{11} \mathrm{~cm}^{-2}$, depending on the spacer width. As shown in Fig. 7, the PL splitting $\Delta(H)$ in such samples is not only exceptionally large but increases in a dramatic way on lowering temperature. Actually, the appearance of zero-field splitting is also visible in Fig. 7. No such effects are detected either in undoped structures or in the presence of illumination by white light that depletes the quantum well from the holes. The phase transition occurs at about $1.8 \mathrm{~K}$ for $x=0.024$ (Fig. 7) [56] and at $c a .2 .5 \mathrm{~K}$ for $x=0.037$ [57]. According to Eq. (1), these magnitudes of $T_{\mathrm{c}}$ imply $A_{\mathrm{F}}=2.5 \pm 0.5$, the value in agreement 


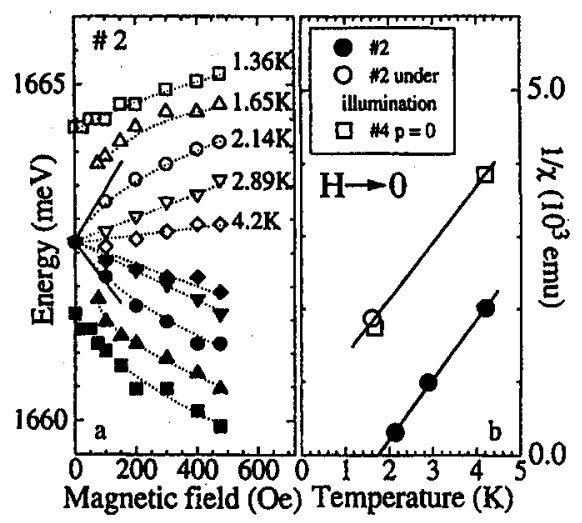

Fig. 7. (a) Energy $E_{ \pm}$of photoluminescence maxima at $\sigma^{+}$and $\sigma^{-}$circular polarizations (full and empty symbols, respectively) as a function of the magnetic field $H$ for selected temperatures in a modulation-doped p-type QW of $\mathrm{Cd}_{0.976} \mathrm{Mn}_{0.024} \mathrm{Te}$. Dotted lines are guides for the eye; solid lines denote the assumed initial slope of $E_{ \pm}$at $2.14 \mathrm{~K}$. (b) Inverse magnetic susceptibility calculated from $\mathrm{d}\left(E_{-}-E_{+}\right) / \mathrm{d} H$ at $H \rightarrow 0$, as given by data in (a) for $p$-type $\mathrm{QW}$ of $\mathrm{Cd}_{0.976} \mathrm{Mn}_{0.024} \mathrm{Te}$ (full circles). The results for empty $\mathrm{Cd}_{0.976} \mathrm{Mn}_{0.024} \mathrm{Te} \mathrm{QWs}$, where antiferromagnetic interactions dominate, are shown by empty symbols. Straight lines show the expected Curie-Weiss dependences (after [56]).

with $A_{F} \equiv 1+g_{3}+g_{4}=2.3$, obtained from the studies of magnetoresistance in $\mathrm{Cd}_{1-x} \mathrm{Mn}_{x}$ Se:In in the weakly localized regime [58].

Particularly strong can be a combined effect of disorder and on-site Hubbard repulsion in the vicinity of the metal-to-insulator transition (MIT) in doped semiconductors. Here, a growing amount of evidences has been collected in favor of a phenomenological two-fluid model of electronic states [59]. According to that model the conversion of itinerant electrons into local moments occurs gradually, and begins already on the metal side of the MIT, leading to the coexistence of the extended and strongly localized states. In magnetic materials, the local moments can polarized, via the $s-d$ interaction, the neighboring Mn spins (there are about $200 \mathrm{Mn}$ ions within the Bohr orbit in $n-\mathrm{Cd}_{0.95} \mathrm{Mn}_{0.05} \mathrm{Se}$ ). The bound magnetic polarons formed in this way not only impose a local ferromagnetic order above $T_{\mathrm{c}}$, but constitute also the centers of efficient spin-dependent scattering for itinerant electrons $[58,60,61]$. The corresponding scattering rate is proportional to the degree of polaron polarization. The latter is proportional to the magnetic susceptibility of the Mn spins, $\chi(T)$, and therefore increases steeply on approaching the ferromagnetic phase transition.

The above mechanism accounts presumably for critical scattering of holes and negative magnetoresistance detected recently in $p-\mathrm{Ga}_{1-x} \mathrm{Mn}_{x}$ As near $T_{\mathrm{c}}$ [49]. By contrast, no such effects are seen in $p-\mathrm{Pb}_{1-x-y} \mathrm{Mn}_{x} \mathrm{Sn}_{y} \mathrm{Te}$ [62], where a large dielectric constant reduces the Hubbard repulsion, and thus precludes the formation of bound magnetic polarons. Actually; the absence of both critical scattering in $p-\mathrm{Pb}_{1-x-y} \mathrm{Mn}_{x} \mathrm{Sn}_{y} \mathrm{Te}$ [62] and red shift of PL in $p-\mathrm{Cd}_{1-x} \mathrm{Mn}_{x} \mathrm{Te}$ quantum wells at $T \geq T_{\mathrm{c}}$ demonstrates the minor importance of thermodynamic fluctuations. 
This substantiates the validity of the MFA in the case of the carrier-induced ferromagnetic transition in semiconductor systems.

\section{Photomagnetization}

Optical studies constitute a well-established tool to probe magnetic semiconductors but equally appealing is the possibility of modifying magnetic properties by light. For instance, the carrier concentration in any modulation-doped QW can be controlled by photons of energy corresponding to the gap in the barrier material [63]. This is due to the existence of a potential barrier between the impurities and the well, which makes that a part of the carriers resides on the impurities in the barrier, not in the QW under stationary illumination. This method was applied [56] in order to turn the $\mathrm{Cd}_{1-x} \mathrm{Mn}_{x} \mathrm{Te} \mathrm{QW}$ from the ferromagnetic to a paramagnetic phase, as shown in Fig. $7 \mathrm{~b}$.

It has been also demonstrated that optical pumping, that is the creation of a spin density of photocarriers by circularly polarized light, results in the appearance of magnetization $m$ of the localized spins [64, 24]. In the framework of the MFA, $m=\chi(T) H^{*}$, where the molecular field $H^{*}$ is proportional to the spin density of the photocarriers. Under stationary conditions, the latter is given by the product of the generation rate and spin-relaxation time $\tau_{\mathrm{s}}$. If scattering by thermodynamic fluctuations of magnetization is the dominant relaxation mechanism of the carrier spins then $\tau_{\mathbf{s}}{ }^{-1} \propto \chi(T) T$, and thus [38] $m \propto 1 / T$. This expectation is corroborated by experimental results presented in Fig. 8 .

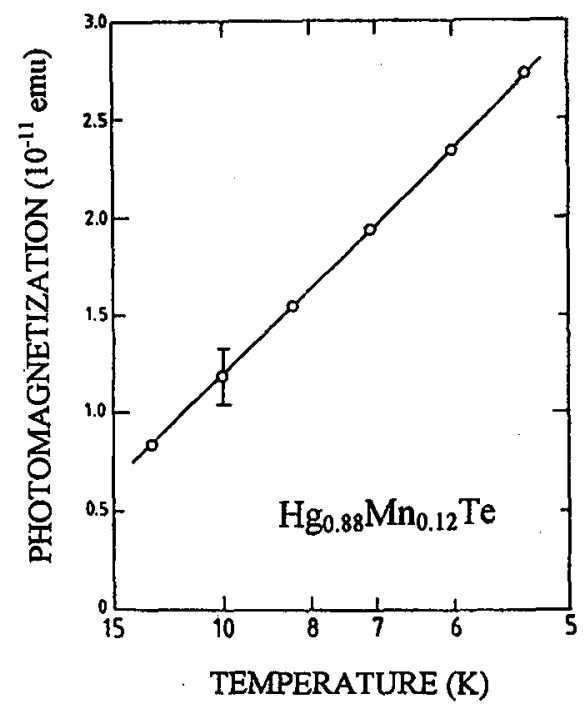

Fig. 8. Magnetization induced by optical pumping showing theoretically expected (solid line) proportionality of the photomagnetization to the inverse temperature (after [38]). 


\section{Summary}

Thought selectively, this review documents a great progress achieved over the recent years in the understanding of the effects of the exchange interaction upon the localized and delocalized carriers in semiconductors containing magnetic constituents. With no doubt, however, feature studies will show how our present understanding would have been incomplete, and how many challenging problems would have been ahead.

\section{Acknowledgments}

I would like to thank my formal and informal co-workers in Warsaw, Linz, and Grenoble for years of fruitful and enjoyable interactions. I am particularly indebted to Günther Bauer for his hospitality during my stay at the Kepler University in Linz, where this paper was prepared.

\section{References}

[1] See, e.g., Diluted Magnetic Semiconductors, Eds. J.K. Furdyna, J. Kossut, in series Semimetals and Semiconductors, Eds. R.K. Willardson, A.C. Beer, Academic Press, New York 1988; Semimagnetic Semiconductors and Diluted Magnetic Semiconductors, Eds. M. Averous, M. Balkanski, Plenum, London 1991; Diluted Magnetic Semiconductors, Ed. M. Jain, World Scientific, Singapu un 1991; T. Dietl, in: Handbook on Semiconductors, 2nd ed., in series Materials, Properties and Preparations, Ed. S. Mahajan, Vol. 3b, North-Holland, Amsterdam 1994, Ch. 17.

[2] P.G. DeGennes, Phys. Rev. 118, 114 (1960); M.A. Krivoglaz, Usp. Fiz. Nauk 111, 617 (1973) [Sov. Phys. Usp. 16, 856 (1974)]; E.L. Nagaev, Physics of Magnetic Semiconductors, Mir, Moscow 1983.

[3] See, P.A. Wolff, in: Diluted Magnetic Semiconductors, Eds. J.K. Furdyna, J. Kossut, in series Semimetals and Semiconductors, Eds. R.K. Willardson, A.C. Beer, Academic Press, New York 1988, p. 413.

[4] D.R. Yakovlev, K.V. Kavokin, Comments Condens. Matter Phys. 18, 51 (1996).

[5] A. Golnik, J. Gaj, M. Nawrocki, R. Planel, C. Benoit à la Guillaume, J. Phys. Soc. Jpn. Suppl. A 49, 819 (1980).

[6] M. Nawrocki, R. Planel, G. Fishman, R.R. Gałazka, Phys. Rev. Lett. 46, 735 (1981).

[7] D.L. Alov, S.I. Gubarev, V.B. Timofeev, Zh. Eksp. Teor. Fiz. 84, 1806 (1983) [Sov. Phys. JETP 57, 1052 (1983)].

[8] D. Heiman, P.A. Wolf, J. Warnock, Phys. Rev. B 27, 4848 (1983).

[9] R. Planel, T.H. Nhung, G. Fishman, M. Nawrocki, J. Phys. (Paris) 45, 1071 (1984).

[10] D.L. Peterson, D.U. Bartholomew, U. Debska, A.K. Ramdas, S. Rodriguez, Phys. Rev. B 32, 323 (1985).

[11] E.D. Isaacs, D. Heiman, M.J. Graf, B.B. Goldberg, R. Kershaw, D. Ridgley, K. Dwight, A. Wold, J. Furdyna, J.S. Brooks, Phys. Rev. B 37, 7108 (1988).

[12] T. Dietl, J. Spałek, Phys. Rev. Lett. 48, 355 (1982); Phys. Rev. B 28, 1548 (1983).

[13] T. Dietl, J. Magn: Magn. Mater. 38, 34 (1983); see also S.M. Ryabchenko, Yu.G. Semenov, Zh. Eksp. Teor. Fiz. 84, 1419 (1983) [Sov. Phys. JETP 57, 8251 (1983)]; D. Heiman et al., Ref. [8]. 
[14] C. Benoit à la Guillaume, Phys. Status Solidi B 175, 369 (1993).

[15] J. Jaroszyński, T. Dietl, M. Sawicki, E. Janik, Physica B 117-118, 473 (1983); also J. Jaroszyński, T. Dietl, Solid State Commun. 55, 491 (1985).

[16] T. Wojtowicz, S. Koleśnik, I. Miotkowski, J.K. Furdyna, Phys. Rev. Lett. 70, 2317 (1993).

[17] A. Golnik, J. Ginter, J.A. Gaj, J. Phys C 16, 6073 (1983).

[18] J.H. Harris, A.V. Nurmikko, Phys. Rev. Lett. 51, 147 (1983).

[19] C.A. Hubert, A.V. Nurmikko, Solid State Commun. 48, 675 (1983); W. Hayes, K.S. Wong, J.F. Ryan, A.K. Ramdas, J. Lumin. 40\&41, 72 (1988); Y. Oka, K. Nakamura, I. Souma, M. Kido, H. Fujisaki, J. Lumin. 38, 263 (1987); H. Akinaga, K. Takita, S. Sasaki, S. Takeyma, N. Miura, T. Nakayama, F. Minami, K. Inoue, Phys. Rev. B 46, 13136 (1992).

[20] J.J. Zayhowski, R.N. Kershaw, D. Ridgley, K. Dwight, A. Wold, R.R. Gałązka, W. Giriat, Phys. Rev. B 35, 6950 (1987).

[21] T. Itoh, E. Komatsu, J. Lumin. 38, 266 (1987).

[22] Y. Oka, K. Ishikawa, I. Souma, M. Nakamura, in: Proc. 20th Int. Conf. on the Physics of Semiconductors, Thessaloniki (Greece) 1990, Eds. E.M. Anastassakis, J.D. Joannopoulos, Word Scientific, Singapore 1990, p. 1939.

[23] G. Mackh, W. Ossau, D.R. Yakovlev, A. Waag, G. Landwehr, R. Hellmann, E.O. Göbel, Phys. Rev. B 50, 14069 (1994).

[24] D.D. Awschalom, J. Warnock, S. von Molnar, Phys. Rev. Lett. 58, 812 (1987).

[25] D.D. Awschalom, J. Warnock, J.M. Hong, L.L. Chang, M.B. Ketchen, W.J. Gallagher, Phys. Rev. Lett. 62, 199 (1989).

[26] D.D. Awschalom, M.R. Freeman, N. Samarth, H. Luo, J.K. Furdyna, Phys. Rev. Lett. 66, 1212 (1991).

[27] D.R. Yakovlev, W. Ossau, G. Landwehr, R.N. Bicknell-Tassius, A. Waag, S. Schmeusser, I.N. Uraltsev, A. Pohlmann, E.O. Göbel, J. Cryst. Growth 117, 854 (1992).

[28] W. Grieshaber, C. Gourgon, P. Lavallard, Y. Merle d'Aubigné, J. Cibert, G. Feuillet, unpublished.

[29] M.R. Freeman, D.D. Awschalom, J.M. Hong, L.L. Chang, Phys. Rev. Lett. 64, 2430 (1990).

[30] T. Dietl, P. Peyla, W. Grieshaber, Y. Merle d'Aubigné, Phys. Rev. Lett. 74, 474 (1995).

[31] D. Scalbert, J. Cernogora, C. Benoit à la Guillaume, Solid State Commun. 66, 571 (1988).

[32] T. Struz, A.M. Witowski, P. Wyder, Phys. Rev. Lett. 68, 3912 (1992); T. Strutz, Ph.D. Thesis, Hartung-Gorre, Konstanz 1991, p. 97.

[33] H.A. Sayad, S. Bhagat, Phys. Rev. B 31, 591 (1985).

[34] R.E. Kremer, J.K. Furdyna, Phys. Rev. B 32, 5591 (1985).

[35] P. Boudinet, G. Bastard, Semicond. Sci. Technol. 8, 1535 (1993).

[36] A.V. Kavokin, K.V. Kavokin, Semicond. Sci. Technol. 8, 191 (1993).

[37] M.A. Butler, S.J. Martin, R.J. Baughman, Appl. Phys. Lett. 49, 1053 (1986).

[38] H. Krenn, K. Kaltenegger, T. Dietl, J. Spałek, G. Bauer, Phys. Rev. B 39, 10918 (1989). 
[39] L. Świerkowski, T. Dietl, Acta Phys. Pol. A 73, 431 (1988); L.R. Ram-Mohan, P.A. Wolff, Phys. Rev. B 38, 1330 (1988).

[40] F. Rys, J.S. Helman, W. Baltensperger, Phys. Kondens. Materie 6, 105 (1967); R.B. Bylsma, W.M. Becker, J. Kossut, U. Debska, D.R. Yoder-Short, Phys. Rev. B 33, 8207 (1986); J.A. Gaj, A. Golnik, Acta Phys. Pol. A 71, 197 (1987).

[41] C. Benoit à la Guillaume, D. Scalbert, T. Dietl, Phys. Rev. B 46, 9853 (1992).

[42] J. Tworzydło, Phys. Rev. B 50, 14591 (1994); Solid State Commun. 94, 821 (1995).

[43] S.I. Gubarev, Fiz. Tverd. Tela 32, 635 (1990) [Sov. Phys.-Solid State 32, 373 (1990)] and references therein.

[44] M. Nawrocki, J.P. Lascaray, D. Coquillat, M. Demianiuk, in: Diluted Magnetic Semiconductors, Eds. R.L. Aggarwal, J.K. Furdyna, S. von Molnar, Materials Research Society, Pittsburgh 1987, p. 65.

[45] M. Herbich, E. Kłopotowski, W. Mac, A. Stachow, A. Twardowski, J. Tworzydło, M. Demianiuk, J. Cryst. Growth 184/185, 992 (1998).

[46] See, P. Łazarczyk, T. Story, M. Arciszewska, R.R. Gałązka, J. Magn. Magn. Mater. 169, 151 (1997) and references therein.

[47] See, S. Koshihara, A. Oiwa, M. Hirasawa, S. Katsumoto, Y. Iye, C. Urano, H. Takagi, H. Munekata, Phys. Rev. Lett. 78, 4617 (1997); H. Ohno, A. Shen, F. Matsukura, A. Oiwa, A. Endo, S. Katsumoto, Y. Iye, Appl. Phys. Lett. 69, 363 (1996).

[48] H. Ohno, F. Matsukura, A. Shen, Y. Sugawara, A. Oiwa, A. Endo, S. Katsumoto, Y. Iye, in: Proc. 23rd Int. Conf. on the Physics of Semiconductors, Berlin 1996, Eds. M. Scheffler, R. Zimmermann, World Scientific, Singapore 1996, p. 405.

[49] F. Matsukura, H. Ohno, A. Shen, Y. Sugawara, Phys. Rev. B 57, R2037 (1998).

[50] J. Blinowski, P. Kacman, J.A. Majewski, Phys. Rev. B 53, 9524 (1996).

[51] T. Wojtowicz, G. Karczewski, J. Kossut, Thin Solid Films 306, 271 (1997).

[52] T. Dietl; A. Haury, Y. Merle d'Aubigné, Phys. Rev. B 55, R3347 (1997).

[53] E.A. Pashitskii, S.M. Ryabchenko, Fiz. Tverd. Tela 21, 545 (1979) [Sov. Phys. Solid State 21, 322 (1979)].

[54] J.A. Gaj, W. Grieshaber, C. Bodin-Deshayes, J. Cibert, G. Feuillet, Y. Merle d'Aubigné, A. Wasiela, Phys. Rev. B 50, 5512 (1994).

[55] B.L. Altshuler, A.G. Aronov, A.Yu. Zyuzin, Pis'ma Zh. Eksp. Teor. Fiz. 38, 128 (1983) [JETP Lett. 38, 153 (1983)].

[56] A. Haury, A. Wasiela, A. Arnoult, J. Cibert, S. Tatarenko, T. Dietl, Y. Merle d'Aubigné, Phys. Rev. Lett. 79, 511 (1997).

[57] J. Cibert, P. Kossacki, A. Haury, A. Wasiela, Y. Merle d'Aubigné, T. Dietl, A. Arnoult, S. Tatarenko, Proc. Int. Conf. II-VI Compounds, Grenoble 1997, J. Cryst. Growth 184/185, 898 (1998).

[58] M. Sawicki, T. Dietl, J. Kossut, J. Igalson, T. Wojtowicz, W. Plesiewicz, Phys. Rev. Lett. 56, 508 (1986).

[59] See, e.g., R.N. Bhatt, M.A. Paalanen, S. Sachdev, J. Phys. (France) 49, C8-1179 (1988).

[60] T. Dietl, M. Sawicki, E.D. Isaacs, M. Dahl, D. Heiman, M.J. Graf, S.I. Gubariev, D.L. Alov, Phys. Rev. B 43, 3154 (1991). 
[61] P. Głód, T. Dietl, M. Sawicki, I. Miotkowski, Physica B 194-196, 995 (1994).

[62] P. Eazarczyk, W. Dobrowolski, T. Story, in: Extended Abstracts of Int. Conf. on Electron Localization and Quantum Transport in Solids, Jaszowiec (Poland) 1996, Ed. T. Dietl, Institute of Physics, Warsaw 1996, p. 129.

[63] A.J. Shields, J.L. Osborne, M.Y. Simmons, D.A. Ritchie, M. Pepper, Semicond. Sci. Technol. 11, 890 (1996) and references therein.

[64] H. Krenn, W. Zawadzki, G. Bauer, Phys. Rev. Lett. 55, 1510 (1985). 